\title{
Women's Awareness and Healthcare Provider Discussions about Zika Virus during Pregnancy, United States, 2016-2017
}

Letitia Williams, Denise V. D’Angelo, Brenda Bauman, Ada C. Dieke, Sascha R. Ellington, Carrie K. Shapiro-Mendoza, Shanna Cox, Philip Hastings, Holly Shulman, Leslie Harrison, Martha Kapaya, Wanda D. Barfield, Lee Warner, PRAMS Zika State Working Group ${ }^{1}$

We surveyed women with a recent live birth who resided in 16 US states and 1 city during the 2016 Zika outbreak. We found high awareness about the risk of Zika virus infection during pregnancy and about advisories to avoid travel to affected areas but moderate levels of discussions with healthcare providers.

7 ika ika virus infection (ZIKV), transmitted primarily by the Aedes aegypti and Ae. albopictus mosquitoes, is a serious threat to pregnant women because of the risk of microcephaly and other birth defects in infants $(1,2)$. In late 2015, an unprecedented ZIKV outbreak emerged in South America and spread rapidly into other parts of the Americas. Although the outbreak has subsided, and pregnant women residing in the United States were at risk for ZIKV infection primarily if they traveled to affected areas or had sexual contact with a partner who traveled to an affected area, information about the outbreak was publicized (3). In particular, travel advisories and guidance about ZIKV were published during the 2016 Zika outbreak (4-6), but the degree of awareness of ZIKV among pregnant women in the United States is unknown. We examined awareness of ZIKV, discussions about ZIKV with healthcare providers, and knowledge of travel advisories to avoid ZIKV-affected areas during pregnancy among women who delivered a live infant during the outbreak.

\section{The Study}

The Pregnancy Risk Assessment Monitoring System (PRAMS) is a state-specific, population-based surveillance system implemented by the Centers for

Author affiliation: Centers for Disease Control and Prevention, Atlanta, Georgia, USA

DOI: https://doi.org/10.3201/eid2605.190727
Disease Control and Prevention (CDC) and state and local health departments to collect information about experiences and behaviors before, during, and after pregnancy among women with a live birth. A stratified random sample is drawn from birth certificate records every month in each participating site. Women are surveyed by mail or telephone 2-6 months after a live birth. Data are weighted to account for the stratified sampling design and to adjust for differential nonresponse (7). We analyzed PRAMS data from 16 US states and 1 city, referred to here as sites (Alabama, Connecticut, Florida, Illinois, Maryland, Massachusetts, Missouri, New Jersey, New York, Pennsylvania, South Carolina, Tennessee, Vermont, Virginia, West Virginia, and Wisconsin, plus New York City), for women who gave birth during March 2016-February 2017.

CDC and participating sites developed supplemental questions on ZIKV in 2016 (8), which sites voluntarily included in their surveys. Once added to the survey, the questions were integrated into the regular PRAMS data collection system, including data processing and weighting, and were considered part of the annual dataset.

We calculated prevalence estimates and 95\% CIs for 3 ZIKV-related outcomes during pregnancy, a subset of the information collected in the supplement: never having heard of ZIKV, talking to a healthcare provider about ZIKV, and having knowledge of ZIKV-related travel advisories during pregnancy. We examined these outcomes by maternal demographics, including age, race/ethnicity, education, marital status, source of payment for delivery, infant birth month, and state of residence.

\footnotetext{
${ }^{1}$ Members of the PRAMS Zika State Working Group are listed at the end of this article.
} 
We used multivariable logistic regression to assess the relationships between maternal demographics and each outcome using adjusted prevalence ratios (aPRs) and 95\% CIs. We adjusted models for all demographics examined, along with factors likely to influence access to healthcare and exposure to information about ZIKV. We completed our analyses using SAS version 9.4 (https:/ / www. sas.com) and SAS-callable SUDAAN 11.0 (https:/ / www.rti.org) software to account for PRAMS complex survey design.

Of 12,845 women sampled from the 17 sites during the study period, 8,711 (68\%) women responded.
Among respondents, most women were 25-34 years of age $(59.7 \%)$, were non-Hispanic white $(56.9 \%)$, had more than a high school education $(65.1 \%)$, were married $(61.3 \%)$, and reported private insurance as a source of payment for delivery (55.8\%) (data not shown). Overall, $8.8 \%$ of women had never heard of ZIKV during their recent pregnancy. These women were more likely to be $<35$ years of age, be non-Hispanic black or of other race, have a high school education or less, be unmarried, and report Medicaid as a source of payment for delivery than women who had heard of ZIKV (Appendix, https:/ / wwwnc.cdc.gov/ EID/article/26/5/19-0727-App1.pdf).

Table 1. Frequency of talking with a healthcare provider about Zika virus and knowledge of CDC travel advisories among women who had heard of Zika virus during their pregnancy and delivered a live infant, 17 US PRAMS sites, March 2016-February 2017*

Women who had heard of Zika virus during pregnancy, $n=7,920$

\begin{tabular}{|c|c|c|c|c|c|c|c|c|}
\hline \multirow{4}{*}{$\begin{array}{l}\text { Maternal } \\
\text { characteristics }\end{array}$} & \\
\hline & \multicolumn{6}{|c|}{ Talked with a healthcare provider about Zika virus } & \multirow{2}{*}{\multicolumn{2}{|c|}{$\begin{array}{c}\text { Had knowledge of CDC } \\
\text { travel advisory }\end{array}$}} \\
\hline & \multicolumn{2}{|r|}{ Total } & \multicolumn{2}{|c|}{ Provider initiated } & \multicolumn{2}{|c|}{ Respondent initiated } & & \\
\hline & No.† & $\%(95 \% \mathrm{Cl}) \ddagger$ & No.† & $\%(95 \% \mathrm{Cl}) \ddagger$ & No.† & $\%(95 \% \mathrm{Cl}) \ddagger$ & No.† & $\%(95 \% \mathrm{Cl}) \ddagger$ \\
\hline Overall & 4,505 & $\begin{array}{c}58.8 \\
(57.3-60.3)\end{array}$ & 2,889 & $\begin{array}{c}63.5 \\
(61.6-65.4)\end{array}$ & 1,616 & $\begin{array}{c}36.4 \\
(34.5-38.3)\end{array}$ & 7,204 & $\begin{array}{c}91.9 \\
(91.0-92.7)\end{array}$ \\
\hline \multicolumn{9}{|l|}{ Age } \\
\hline$\leq 24$ & 801 & $\begin{array}{c}58.1 \\
(54.5-61.7)\end{array}$ & 600 & $\begin{array}{c}74.6 \\
(70.2-79.0)\end{array}$ & 201 & $\begin{array}{c}25.4 \\
(21.0-29.8)\end{array}$ & 1,293 & $\begin{array}{c}89.5 \\
(87.4-91.6)\end{array}$ \\
\hline $25-34$ & 2,707 & $\begin{array}{c}58.6 \\
(56.7-60.6)\end{array}$ & 1,663 & $\begin{array}{c}60.8 \\
(58.3-63.3)\end{array}$ & 1,044 & $\begin{array}{c}39.2 \\
(36.7-41.7)\end{array}$ & 4,315 & $\begin{array}{c}92.1 \\
(91.0-93.3)\end{array}$ \\
\hline$\geq 35$ & 993 & $\begin{array}{c}60.3 \\
(57.2-63.5)\end{array}$ & 622 & $\begin{array}{c}61.2 \\
(57.2-65.3)\end{array}$ & 371 & $\begin{array}{c}38.8 \\
(34.7-42.8)\end{array}$ & 1,580 & $\begin{array}{c}93.8 \\
(92.3-95.3) \\
\end{array}$ \\
\hline \multicolumn{9}{|l|}{ Race/ethnicity } \\
\hline White, non-Hispanic & 2,314 & $\begin{array}{c}59.1 \\
(57.1-61.1)\end{array}$ & 1,365 & $\begin{array}{c}58.7 \\
(56.1-61.3)\end{array}$ & 949 & $\begin{array}{c}41.3 \\
(38.7-43.9)\end{array}$ & 3,794 & $\begin{array}{c}94.1 \\
(93.1-95.0)\end{array}$ \\
\hline Black, non-Hispanic & 837 & $\begin{array}{c}60.3 \\
(56.4-64.1)\end{array}$ & 596 & $\begin{array}{c}71.9 \\
(67.3-76.5)\end{array}$ & 241 & $\begin{array}{c}28.1 \\
(23.5-32.7)\end{array}$ & 1,285 & $\begin{array}{c}87.8 \\
(85.1-90.5)\end{array}$ \\
\hline Hispanic & 800 & $\begin{array}{c}57.4 \\
(53.6-61.1)\end{array}$ & 600 & $\begin{array}{c}74.0 \\
(69.7-78.4)\end{array}$ & 200 & $\begin{array}{c}26.0 \\
(21.6-30.3)\end{array}$ & 1,233 & $\begin{array}{c}89.8 \\
(87.6-92.1)\end{array}$ \\
\hline Other, non-Hispanic & 525 & $\begin{array}{c}57.9 \\
(53.3-62.4)\end{array}$ & 313 & $\begin{array}{c}62.2 \\
(56.1-68.2) \\
\end{array}$ & 212 & $\begin{array}{c}37.8 \\
(31.8-43.9) \\
\end{array}$ & 838 & $\begin{array}{c}88.6 \\
(85.7-91.5)\end{array}$ \\
\hline \multicolumn{9}{|l|}{ Education } \\
\hline $\begin{array}{l}\text { High school } \\
\text { or below }\end{array}$ & 1,229 & $\begin{array}{c}53.1 \\
(50.1-56.1)\end{array}$ & 921 & $\begin{array}{c}73.9 \\
(70.3-77.5)\end{array}$ & 308 & $\begin{array}{c}26.1 \\
(22.5-29.7)\end{array}$ & 2,047 & $\begin{array}{c}86.8 \\
(84.8-88.8)\end{array}$ \\
\hline $\begin{array}{l}\text { Less than high } \\
\text { school }\end{array}$ & 3,228 & $\begin{array}{c}61.5 \\
(59.7-63.3)\end{array}$ & 1,936 & $\begin{array}{c}59.3 \\
(57.0-61.6)\end{array}$ & 1,292 & $\begin{array}{c}40.7 \\
(38.4-43.0)\end{array}$ & 5,075 & $\begin{array}{c}94.4 \\
(93.5-95.2)\end{array}$ \\
\hline \multicolumn{9}{|l|}{ Marital status } \\
\hline Married & 2,942 & $\begin{array}{c}61.5 \\
(59.7-63.3)\end{array}$ & 1,742 & $\begin{array}{c}57.9 \\
(55.5-60.3)\end{array}$ & 1,200 & $\begin{array}{c}42.1 \\
(39.7-44.5)\end{array}$ & 4,655 & $\begin{array}{c}93.9 \\
(93.0-94.8)\end{array}$ \\
\hline Other & 1,558 & $\begin{array}{c}54.3 \\
(51.6-57.1) \\
\end{array}$ & 1,142 & $\begin{array}{c}74.7 \\
(71.6-77.9) \\
\end{array}$ & 416 & $\begin{array}{c}25.3 \\
(22.1-28.4) \\
\end{array}$ & 2,531 & $\begin{array}{c}88.5 \\
(86.8-90.2) \\
\end{array}$ \\
\hline \multicolumn{9}{|c|}{ Source of payment for delivery } \\
\hline Private & 2,738 & $\begin{array}{c}62.5 \\
(60.6-64.4)\end{array}$ & 1,606 & $\begin{array}{c}58.3 \\
(55.8-60.8)\end{array}$ & 1,132 & $\begin{array}{c}41.7 \\
(39.2-44.2)\end{array}$ & 4,208 & $\begin{array}{c}94.1 \\
(93.1-95.0)\end{array}$ \\
\hline Medicaid & 1,564 & $\begin{array}{c}54.2 \\
(51.6-56.8)\end{array}$ & 1,135 & $\begin{array}{c}72.0 \\
(68.9-75.2)\end{array}$ & 429 & $\begin{array}{c}28.0 \\
(24.8-31.1)\end{array}$ & 2,629 & $\begin{array}{c}89.0 \\
(87.5-90.6)\end{array}$ \\
\hline No insurance & 113 & $\begin{array}{c}46.7 \\
(37.4-56.1) \\
\end{array}$ & 91 & $\begin{array}{c}77.5 \\
(65.7-89.4)\end{array}$ & 22 & $\begin{array}{c}22.5 \\
(10.6-34.3) \\
\end{array}$ & 206 & $\begin{array}{c}85.4 \\
(78.0-92.8) \\
\end{array}$ \\
\hline \multicolumn{9}{|l|}{ Infant birth month } \\
\hline 2016 Mar-Aug & 1,778 & $\begin{array}{c}53.5 \\
(51.1-55.9)\end{array}$ & 1,087 & $\begin{array}{c}61.9 \\
(58.7-65.2)\end{array}$ & 691 & $\begin{array}{c}38.1 \\
(34.8-41.3)\end{array}$ & 3,131 & $\begin{array}{c}90.7 \\
(89.2-92.1)\end{array}$ \\
\hline 2016 Sep-2017 Feb & 2,723 & $\begin{array}{c}63.1 \\
(61.2-65.1)\end{array}$ & 1,798 & $\begin{array}{c}64.6 \\
(62.3-67.0)\end{array}$ & 925 & $\begin{array}{c}35.4 \\
(33.0-37.7)\end{array}$ & 4,057 & $\begin{array}{c}93.0 \\
(92.0-94.0)\end{array}$ \\
\hline $\begin{array}{l}\text { *Data aggregated for } 17 \mathrm{si} \\
\text { Pennsylvania, South Carol } \\
\text { Pregnancy Risk Assessme } \\
\text { †Unweighted. } \\
\text { †Weighted. }\end{array}$ & 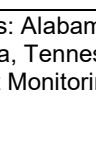 & ystem. & & & & ri, New Jersey, & & $\begin{array}{l}\text { York City, } \\
\text { ention; PRAMS, }\end{array}$ \\
\hline
\end{tabular}


Table 2. Factors associated with never having heard of Zika virus, talking with a healthcare provider, and knowledge of CDC travel advisories among women who delivered a live infant, 17 US PRAMS sites, March 2016-February $2017^{*}$

\begin{tabular}{|c|c|c|c|}
\hline \multirow[b]{3}{*}{ Maternal characteristic } & \multicolumn{3}{|c|}{ Adjusted prevalence ratio $(95 \% \mathrm{Cl}) \dagger$} \\
\hline & \multirow[b]{2}{*}{$\begin{array}{c}\text { Women who had never heard } \\
\text { of Zika virus, } n=791\end{array}$} & \multicolumn{2}{|c|}{ Women who had heard of Zika virus during pregnancy } \\
\hline & & $\begin{array}{c}\text { Talked with a healthcare provider } \\
\text { about Zika virus, } n=4,505\end{array}$ & $\begin{array}{l}\text { Had knowledge of CDC } \\
\text { travel advisory, } n=7,204\end{array}$ \\
\hline \multicolumn{4}{|l|}{ Age } \\
\hline$\leq 24$ & $1.77(1.28-2.44)$ & $1.07(0.98-1.17)$ & $1.00(0.97-1.02)$ \\
\hline$\overline{2} 5-34$ & $1.22(0.92-1.62)$ & $0.99(0.93-1.06)$ & 0.99 (0.96-1.01) \\
\hline$\geq 35$ & Referent & Referent & Referent \\
\hline \multicolumn{4}{|l|}{ Race/ethnicity } \\
\hline White, non-Hispanic & Referent & Referent & Referent \\
\hline Black, non-Hispanic & $1.86(1.46-2.37)$ & $1.12(1.04-1.20)$ & $0.97(0.94-1.00)$ \\
\hline Hispanic & $0.69(0.49-0.99)$ & $1.08(1.00-1.17)$ & $0.99(0.97-1.02)$ \\
\hline Other, non-Hispanic & $2.41(1.85-3.13)$ & $0.96(0.87-1.05)$ & $0.92(0.89-0.96)$ \\
\hline \multicolumn{4}{|l|}{ Education } \\
\hline High school or below & $2.22(1.68-2.92)$ & $0.91(0.84-0.97)$ & $0.94(0.92-0.97)$ \\
\hline More than high school & Referent & Referent & Referent \\
\hline \multicolumn{4}{|l|}{ Marital status } \\
\hline Married & Referent & Referent & Referent \\
\hline Other & $1.5(1.19-1.89)$ & $0.92(0.85-0.98)$ & $0.98(0.95-1.00)$ \\
\hline \multicolumn{4}{|c|}{ Source of payment for delivery } \\
\hline Private & Referent & Referent & Referent \\
\hline Medicaid & $1.45(1.3-1.88)$ & $0.88(0.82-0.94)$ & $0.98(0.95-1.00)$ \\
\hline None & $2.46(1.62-3.74)$ & $0.77(0.2-0.96)$ & $0.95(0.88-1.02)$ \\
\hline \multicolumn{4}{|l|}{ Infant birth month } \\
\hline 2016 Mar-Aug & Referent & Referent & Referent \\
\hline 2016 Sep-2017 Feb & $0.81(0.67-0.98)$ & $1.19(1.13-1.26)$ & $1.02(1.00-1.04)$ \\
\hline \multicolumn{4}{|c|}{$\begin{array}{l}\text { *Data aggregated for } 17 \text { sites: Alabama, Connecticut, Florida, Illinois, Maryland, Massachusetts, Missouri, New Jersey, New York, New York City, } \\
\text { Pennsylvania, South Carolina, Tennessee, Vermont, Virginia, West Virginia, and Wisconsin. Final sample sizes varied for each model because of missing } \\
\text { responses. Boldface type indicates statistical significance. CDC, Centers for Disease Control and Prevention; PRAMS, Pregnancy Risk Assessment } \\
\text { Monitoring System. } \\
\dagger \text { All prevalence ratio estimates adjusted for maternal age, race/ethnicity, education, marital status, delivery payment source, infant month of birth, and } \\
\text { state of residence. }\end{array}$} \\
\hline
\end{tabular}

Focusing on the subgroup of women who had heard about ZIKV during their pregnancy, we found that more than half $(58.8 \%)$ reported talking to a healthcare provider about ZIKV. Nearly two thirds $(63.5 \%)$ reported that their provider initiated the conversation; the remaining third $(36.4 \%)$ reported that they initiated the conversation themselves (Table 1). Compared with non-Hispanic white women, non-Hispanic black women were more likely to have talked with a healthcare provider about ZIKV (aPR 1.12 , 95\% CI 1.04-1.20), as were women who gave birth during September 2016-February 2017 compared with those who gave birth in earlier months (aPR 1.19, 95\% CI 1.13-1.26). However, women with a high school education or less (aPR 0.91, 95\% CI 0.84-0.97), women who were not married (aPR 0.92, 95\% CI 0.85-0.98), and women reporting Medicaid (aPR $0.88,95 \%$ CI $0.82-0.94$ ) or no insurance (aPR $0.77,95 \%$ CI $0.2-0.96$ ) at delivery were less likely to have talked with their healthcare provider about ZIKV (Table 2).

Most (91.9\%) women reported knowledge of CDC travel advisories to avoid areas affected by Zika while pregnant (Table 1). Respondents reporting other non-Hispanic race versus non-Hispanic white women were less likely to have knowledge of the travel advisories (aPR 0.92, 95\% CI 0.89-0.96), as were those with a high school education or less compared with women with more than a high school education (aPR 0.94, 95\% CI 0.92-0.97) (Table 2).

In the adjusted analysis, women $\leq 24$ years old were more likely not to have heard of ZIKV compared with women $\geq 35$ years old (aPR 1.77, 95\% CI 1.282.44 ), as were non-Hispanic black women compared with non-Hispanic white women (aPR 1.86, 95\% CI 1.46-2.37) and non-Hispanic women of other races compared with non-Hispanic white women (aPR 2.41, 95\% CI 1.85-3.13). In contrast, Hispanic women were more likely to have heard of ZIKV. Women with a high school education or less, women whose deliveries were paid for by Medicaid, and those who were uninsured at delivery were less likely to have heard of ZIKV compared with their counterparts with more than a high school education and private health insurance (Table 2).

\section{Conclusions}

These findings highlight interactions between pregnant women and their healthcare providers in 17 sites during the height of the 2016 Zika outbreak. Awareness of ZIKV was found to be high, as was awareness of CDC travel advisories to avoid 
travel to Zika-affected areas during pregnancy (both $>90 \%$ ). This awareness was likely obtained from multiple sources, given that only half of women who heard of ZIKV reported discussing it with their healthcare provider during pregnancy. Similar results have been reported in other studies $(9,10)$. Even though awareness was high, disparities existed, related to the smaller proportion of Hispanic and non-Hispanic black women who reported initiating discussions with providers about ZIKV. These differences suggest the opportunity to promote patient advocacy so that patients of all backgrounds feel comfortable asking about key topics if they are not raised by the provider, especially in the case of public health threats.

Our assessment is not without limitations. Data represent only women who recently gave birth to live infants in the 17 sites included in this analysis. Women seen in practices that conducted screening for travel history before a woman talked to her provider may not have reported counseling, especially if they were determined to be at low risk during the screening. Information is self-reported by the mother 2-6 months following the birth of her infant and may be subject to recall and social desirability bias.

PRAMS is the largest state- and populationbased surveillance system in the United States that samples women who delivered live infants. PRAMS was augmented to collect timely data regarding patient and provider interactions related to ZIKV. Information from this analysis can fill data gaps and address the need to understand interactions between pregnant women and their healthcare providers regarding ZIKV.

Members of the PRAMS Zika State Working Group: Fu Zhao, Alabama Department of Public Health; Jennifer Morin, Connecticut Department of Health; Elizabeth C. Stewart, Florida Department of Health; Julie Doetsch, Illinois Department of Health; Laurie Kettinger, Maryland Department of Health; Emily Lu, Massachusetts Department of Health; Sharon Smith Cooley, New Jersey Department of Health; Eirian Coronado, New Mexico Department of Health; Anne Radigan-Garcia, New York State Department of Health; Pricila Mullachery, New York City Department of Health; Sara E. Thuma, Pennsylvania Department of Health; Harley T. Davis, South Carolina Department of Health and Environmental Control; Angiezel Merced-Morales, Tennessee Department of Health; John Davy, Vermont Department of Health; Sara Varner, Virginia Department of Health; Melissa Baker, West Virginia Department of Health; Fiona Weeks, Wisconsin Department of Health Services

\section{About the Author}

Ms. Williams is an epidemiologist with the Women's Health and Fertility Branch, Division of Reproductive Health, National Center for Chronic Disease Prevention and Health Promotion, Centers for Disease Control and Prevention, Atlanta, GA, USA. Her research interests include perinatal surveillance and maternal and child health disparities.

\section{References}

1. Rasmussen SA, Jamieson DJ, Honein MA, Petersen LR. Zika virus and birth defects - reviewing the evidence for causality. N Engl J Med. 2016;374:1981-7. https:/ / doi.org/ 10.1056/NEJMsr1604338

2. Oster AM, Brooks JT, Stryker JE, Kachur RE, Mead P, Pesik NT, et al. Interim guidelines for prevention of sexual transmission of Zika virus - United States, 2016. MMWR Morb Mortal Wkly Rep. 2016;65:120-1. https:/ / doi.org/ 10.15585/mmwr.mm6505e1

3. Hall V, Walker WL, Lindsey NP, Lehman JA, Kolsin J, Landry K, et al. Update: noncongenital Zika virus disease cases - 50 U.S. states and the District of Columbia, 2016. MMWR Morb Mortal Wkly Rep. 2018;67:265-9. https:/ / doi.org/10.15585/mmwr.mm6709a1

4. Centers for Disease Control and Prevention. CDC issues interim travel guidance related to Zika virus for 14 countries and territories in Central and South America and the Caribbean [cited 2019 Aug 6]. https:/ / www.cdc.gov/ media/releases/2016/s0315-zika-virus-travel.html

5. Petersen EE, Staples JE, Meaney-Delman D, Fischer M, Ellington SR, Callaghan WM, et al. Interim guidelines for pregnant women during a Zika virus outbreak - United States, 2016. MMWR Morb Mortal Wkly Rep. 2016;65:30-3. https://doi.org/10.15585/mmwr.mm6502e1

6. Petersen EE, Meaney-Delman D, Neblett-Fanfair R, Havers F, Oduyebo T, Hills SL, et al. Update: interim guidance for preconception counseling and prevention of sexual transmission of Zika virus for persons with possible Zika virus exposure-United States, September 2016. MMWR Morb Mortal Wkly Rep. 2016;65:1077-81. https:/ / doi.org/ 10.15585/mmwr.mm6539e1

7. Shulman HB, D'Angelo DV, Harrison L, Smith RA, Warner L. The Pregnancy Risk Assessment Monitoring System (PRAMS): overview of design and methodology. Am J Public Health. 2018;108:1305-13. https:/ / doi.org/ 10.2105/ AJPH.2018.304563

8. Centers for Disease Control and Prevention. PRAMS Zika supplement, 2016-2017 [cited 2019 Aug 6]. https://www.cdc.gov/prams/special-projects/zika/map/ prams-zika-supplement.htm

9. Guo F, Norton AR, Fuchs EL, Hirth JM, Garcia-Blanco MA, Berenson AB. Provider-patient communication about Zika during prenatal visits. Prev Med Rep. 2017;7:26-9. https://doi.org/10.1016/j.pmedr.2017.05.003

10. Berenson AB, Trinh HN, Hirth JM, Guo F, Fuchs EL, Weaver SC. Knowledge and prevention practices among US immigrants from Zika virus outbreak areas. Am J Trop Med Hyg. 2017;97:155-62. https:// doi.org/10.4269/ajtmh.17-0062

Address for correspondence: Letitia Williams, Centers for Disease Control and Prevention, 4770 Buford Hwy NE, Mailstop F-74, Atlanta, GA 30341-3724, USA; email: LMWilliams@cdc.gov 